\title{
Spin Singlet State in Heptamers Emerging in Spinel Oxide $\mathrm{AlV}_{2} \mathrm{O}_{4}$
}

\author{
Keisuke Matsuda*, Nobuo Furukawa and Yukitoshi Motome $^{1}$ \\ Department of Physics and Mathematics, Aoyama Gakuin University, Fuchinobe 5-10-1, Sagamihara, \\ Kanagawa 229-8558 \\ ${ }^{1}$ Department of Applied Physics, University of Tokyo, Hongo 7-3-1, Bunkyo-ku, Tokyo 113-8656
}

\begin{abstract}
We present our theoretical results on the electronic state in vanadium spinel oxide $\mathrm{AlV}_{2} \mathrm{O}_{4}$. The material is a mixed-valent system with the average valence $\mathrm{V}^{2.5+}$, and $\mathrm{V}$ cations constitute a frustrated pyrochlore structure. It shows a structural transition at $\sim 700 \mathrm{~K}$, leading to the formation of seven V-sites clusters - heptamers. We study the electronic state of the heptamer by explicitly taking account of orbital degree of freedom as well as electron correlations. We show that the ground state of the heptamer for realistic parameters becomes spin-singlet because of strong $\sigma$-type bonding states of $t_{2 g}$ orbitals. The temperature dependence of the magnetic susceptibility in experiments is naturally understood by this singlet formation in heptamers. Our results indicate that in $\mathrm{AlV}_{2} \mathrm{O}_{4}$ orbital physics is relevant to stabilize a cluster-type singlet state instead of a previously proposed charge-ordered state with valence skipping.
\end{abstract}

KEYWORDS: $\mathrm{AIV}_{2} \mathrm{O}_{4}$, spinel, pyrochlore lattice, heptamer, spin singlet, exact diagonalization, multiorbital Hubbard model

\section{Introduction}

Geometrical frustration in strongly correlated systems has attracted much interest for decades. Many well-studied examples are found in frustrated antiferromagnets, where frustration results in nearly degenerate ground-state manifolds of a large number of different spin configurations. $^{1,2}$ In some cases, the degeneracy remains down to the lowest temperature and leads to exotic phenomena such as a liquid state and a glassy state. In general, however, nature does not favor the degeneracy, and tries to find a way of lifting it and to select a unique, nondegenerate ground state. It is very intriguing to clarify the mechanism of lifting the degeneracy.

The pyrochlore lattice is a typical frustrated structure in three dimensions, and the magnets on this lattice structure have attracted much attention because of their severe frustration. The pyrochlore lattice consists of a network of corner-sharing tetrahedra, and is regarded as a three-dimensional analogue of the Kagomé lattice. In fact, the pyrochlore lattice can be viewed as an alternative stacking of Kagomé and triangular planes as shown in Fig. 1. This lattice structure is found in many real compounds, for instance, in spinels, pyrochlore compounds

*E-mail address: corpse@phys.aoyama.ac.jp 


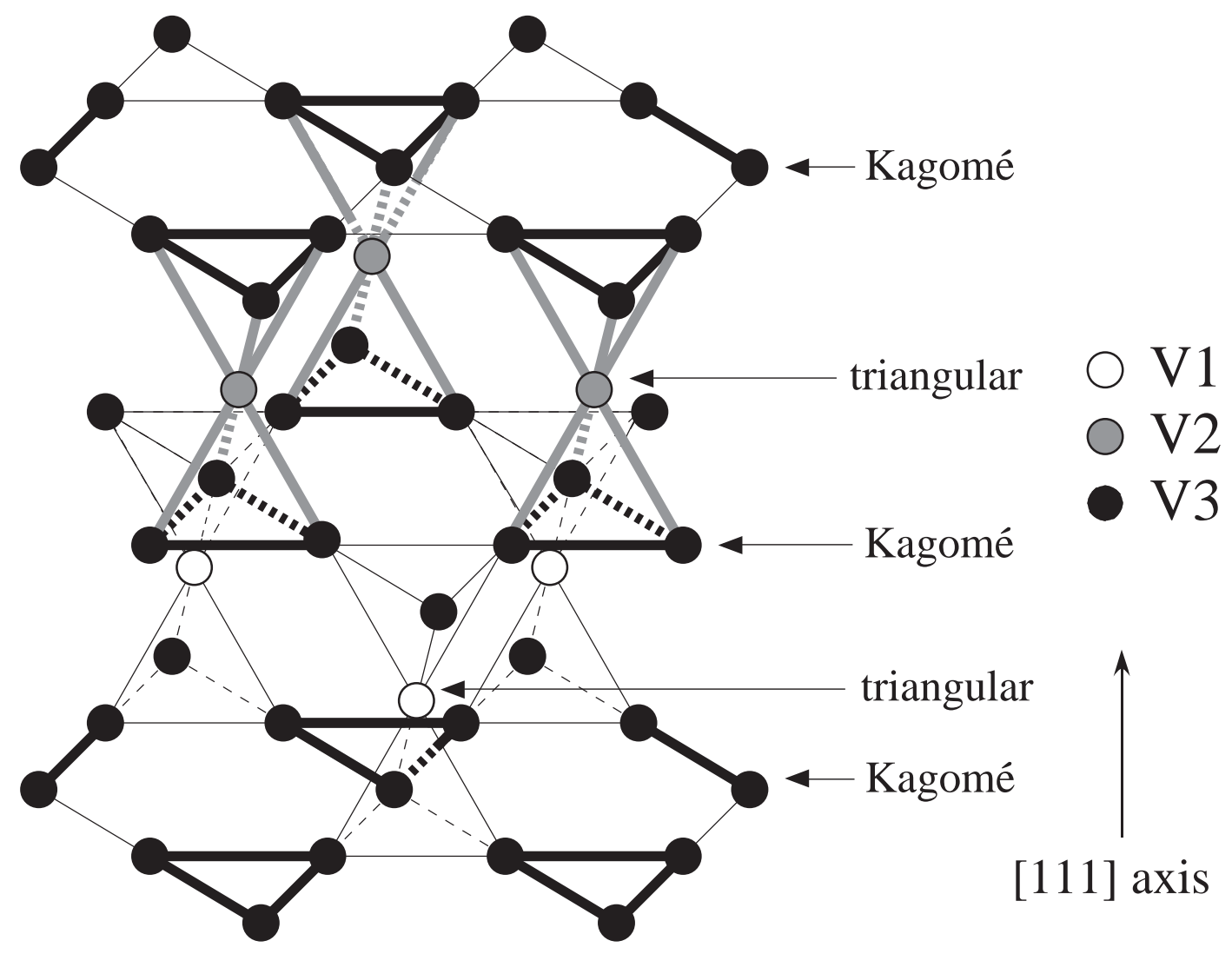

Fig. 1. Schematic picture of the lattice structure of $\mathrm{AlV}_{2} \mathrm{O}_{4}$. For simplicity, only V sites which constitute the pyrochlore structure are shown. There are three crystarographycally inequivalent $\mathrm{V}$ sites (V1, V2 and V3), and V-V bond lengths are substantially different below the structural transition temperature $T_{\mathrm{c}} \sim 700 \mathrm{~K}$. Trimers are shown by bold lines. Two trimers sandwiches a V2 site (connected by gray lines), forming a heptamer. See the text for details.

and cubic Laves-phase intermetallic compounds.

One of the most fundamental problems in the frustrated pyrochlore systems was studied by Anderson. ${ }^{3}$ He examined the ground state and its degeneracy in the Ising model on the pyrochlore lattice, which mimics a possible charge-ordering phenomenon in magnetite $\mathrm{Fe}_{3} \mathrm{O}_{4}$, i.e., the so-called Verwey transition. ${ }^{4}$ He pointed out that the ground state of this model does not exhibit any long-range order and suffers from macroscopic degeneracy. This shows that the frustration may give rise to nontrivial phenomena even in charge degree of freedom. Recently, this 'charge frustration' has also been an issue in several other mixed-valent materials such as $\mathrm{CuIr}_{2} \mathrm{~S}_{4}{ }^{5}$ and $\mathrm{LiV}_{2} \mathrm{O}_{4} \cdot{ }^{6,7}$

A spinel oxide $\mathrm{AlV}_{2} \mathrm{O}_{4}$ is a typical system where this charge-frustration phenomenon is addressed. In this compound, vanadium cations form the pyrochlore lattice and their average valence is $2.5+$. The vanadium cations locate at the octahedral positions, and therefore, fivefold $d$ levels of vanadium are split by the crystal field into low-energy three-fold $t_{2 g}$ levels and 
high-energy two-fold $e_{g}$ levels. Hence, we have $2.5 d$ electrons in the three-fold $t_{2 g}$ levels on average. Because the $t_{2 g}$ levels are partially filled, orbital degree of freedom is also relevant in this compound in addition to charge and spin degrees of freedom. Therefore, $\mathrm{AlV}_{2} \mathrm{O}_{4}$ is an interesting system to study the effects of geometrical frustration and the roles of charge, spin and orbital degrees of freedom.

$\mathrm{AlV}_{2} \mathrm{O}_{4}$ shows a phase transition at $T_{\mathrm{c}} \sim 700 \mathrm{~K}$. At this temperature, a structural change is observed from the high-temperature cubic phase to the low-temperature rhombohedral phase accompanied by a doubling of the unit cell along the [111] direction. ${ }^{8,9}$ This transition was interpreted as a charge ordering with valence skipping in which the charge distribution is differentiated between two different [111] planes, namely, Kagomé and triangular planes in Fig. 1; it is supposed that $\mathrm{V}$ cations in Kagomé layers take the valence $2+$ and those in triangular layers take $4+$. However, there remain two unresolved issues in this charge-ordering scenario. One is the discrepancy between the periodicity of the lattice structure and the charge order. The lattice unit cell is doubled in the [111] direction, which is twice as long as that of charge order. The other unresolved issue is the temperature dependence of the magnetic susceptibility. The susceptibility shows a sudden drop at $T_{\mathrm{c}}$ followed by a Curie-like component in the low-temperature phase. It is difficult to explain this temperature dependence on the basis of the charge-ordering scenario.

Recently, the lattice structure of this compound was reinvestigated. ${ }^{10}$ It was found that below $T_{\mathrm{c}}$, there is an additional superlattice structure within the Kagomé planes, i.e., a 'trimer' formation as shown in Fig. 1. The trimers are paired between neighboring Kagomé planes, which leads to the doubling of the unit cell along the [111] direction. As a result, there are three crystallographically inequivalent $\mathrm{V}$ sites as shown in Fig. 1. Bond lengths between $\mathrm{V}$ sites are estimated as 3.04, 2.81, 2.61 and $3.14 \stackrel{\AA}{A}$ for V1-V3, V2-V3, short V3-V3 (within trimers) and long V3-V3 (between trimers) bonds, respectively. ${ }^{10}$

These substantial differences among the bond lengths imply a decomposition of the whole pyrochlore lattice into two components; one is 'heptamer', i.e., a cluster with seven V sites (one V2 site and six V3 sites) connected by bold and gray lines in Fig. 1, and the other is a remaining V1 site. This decomposition and the heptamer formation may provide an alternative scenario to the previous charge-ordering one, which we call the heptamer scenario hereafter. The formation of the heptamer itself naturally explains the doubling of the unit cell along the [111] direction. The peculiar temperature dependence of the susceptibility may also be compromised if one assumes spin-singlet states in the heptamers and local moments at each V1 sites; the former leads to a sudden drop at $T_{\mathrm{c}}$ and the latter gives rise to the Curie-like component below $T_{\mathrm{c}}$. In fact, it was pointed out that the latter Curie-like component is well fitted by assuming an $S=1$ local moment at each V1 site. $^{10}$

From a theoretical point of view, two important issues must be addressed to understand 
the physical properties of $\mathrm{AlV}_{2} \mathrm{O}_{4}$ on the basis of the heptamer scenario. (i) Why are the heptamers stabilized in this frustrated pyrochlore system? (ii) How does the spin-singlet state emerge in each heptamer? To answer the first question, it is necessary to consider charge, spin and orbital degrees of freedom in the entire pyrochlore lattice, which is very complicated. The second question is also nontrivial. There are in total $18 d$ electrons in each heptamer when we assign $2 d$ electrons to each V1 site to form the $S=1$ moment for the Curie-like contribution. It is not trivial whether we obtain a spin-singlet state with 18 electrons on the seven-site cluster when we take account of the charge, spin and orbital states as well as electron correlations.

In this paper, we will investigate the second problem, that is, the mechanism of spin-singlet formation, by studying the electronic state of one heptamer explicitly. We will discuss the effects of orbital-dependent transfer integrals, electronic correlations and the crystal field, and clarify the parameter region where the spin-singlet ground state is obtained. This systematic study provides a simple physical picture of the spin-singlet formation in the heptamer. We believe that the stabilization mechanism of the spin-singlet state may give a hint for the first question as well.

This paper is organized as follows. In $\S 2$, we introduce a heptamer Hamiltonian on the basis of a multiorbital Hubbard model. Two different singlet states in the noninteracting case are discussed in $\S 3$. In $\S 4$, we show our numerical results for the electronic state of the heptamer Hamiltonian. Section 5 is devoted for the summary and concluding remarks.

\section{Heptamer Hamiltonian}

To consider the electronic state of one heptamer, we start from a multiorbital Hubbard model with three-fold $t_{2 g}$ orbital degeneracy. The Hamiltonian consists of three terms as

$$
\mathcal{H}_{\text {full }}=\mathcal{H}_{\text {kin }}+\mathcal{H}_{\text {int }}+\mathcal{H}_{\text {trig }}
$$

where $\mathcal{H}_{\text {kin }}$ is the kinetic term determined by electron hoppings between $\mathrm{V}$ sites, $\mathcal{H}_{\text {int }}$ represents the on-site Coulomb interactions, and $\mathcal{H}_{\text {trig }}$ is for the crystal-field splitting of $t_{2 g}$ levels induced by the trigonal distortion of $\mathrm{VO}_{6}$ octahedra. The Hamiltonian is defined on the seven-site cluster in Fig. 2(a), and contains 18 electrons in total as mentioned in $\S 1$.

The first term $\mathcal{H}_{\text {kin }}$ is given in the form

$$
\mathcal{H}_{\text {kin }}=-\sum_{\langle i j\rangle} \sum_{\gamma, \gamma^{\prime}} \sum_{\tau} t_{i j}^{\gamma \gamma^{\prime}}\left(c_{i \gamma \tau}^{\dagger} c_{j \gamma^{\prime} \tau}+\text { H.c. }\right),
$$

where $i, j$ and $\tau(=\uparrow, \downarrow)$ are site and spin indices, respectively, and $\gamma, \gamma^{\prime}=1\left(d_{x y}\right), 2\left(d_{y z}\right)$ and $3\left(d_{z x}\right)$ are orbital indices. The site indices take the values from 0 to 6 as shown in Fig. 2(a), and the summation $\langle i j\rangle$ is taken over the nearest-neighbor sites. (At this stage, we neglect the difference between the bond lengths of V2-V3 bonds and V3-V3 bonds in the heptamer.) The heptamer originally consists of the edge-sharing network of $\mathrm{VO}_{6}$ octahedra 
(a)

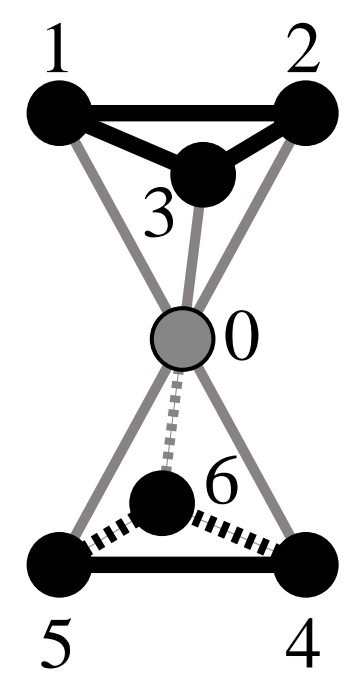

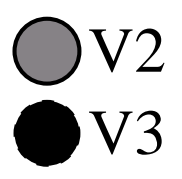

(b)

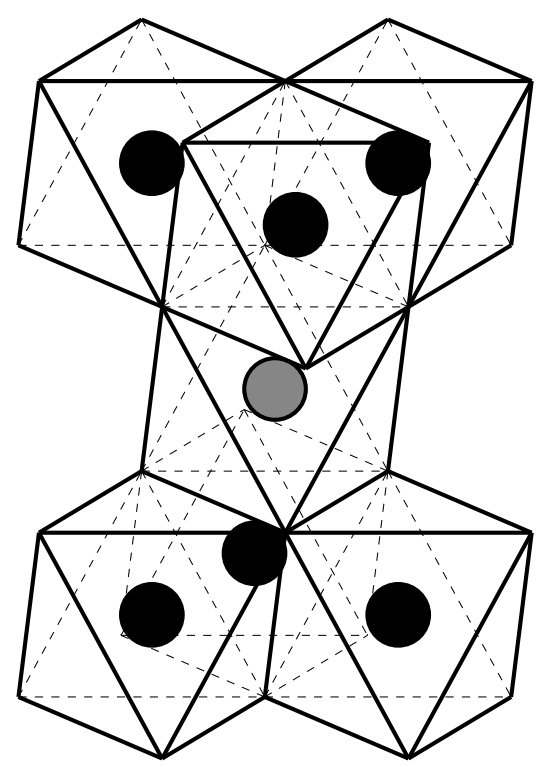

(c)

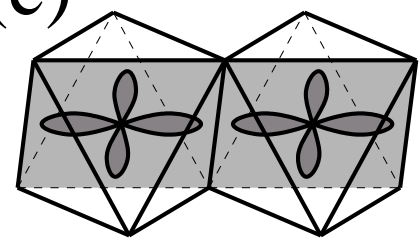

(d)

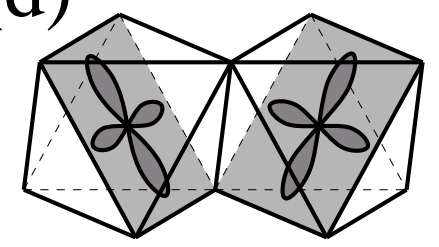

(e)

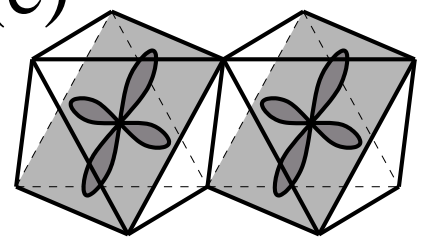

Fig. 2. (a) One heptamer. The site numbering used in our model is shown. (b) Edge-sharing network of $\mathrm{VO}_{6}$ octahedra for the heptamer. Oxygen atoms are at the corners of octahedra. Transfer integrals between $t_{2 g}$ orbitals of (c) $\sigma^{-}$, (d) $\pi_{1^{-}}$and (e) $\pi_{2}$-type transfer integrals, respectively.

as shown in Fig. 2(b). For the edge-sharing configuration, the $d$ - $d$ transfer integrals $t_{i j}^{\gamma \gamma^{\prime}}$ have three different elements as shown in Figs. 2(c)-(e). Here, we call them the $\sigma_{-}, \pi_{1}$ - and $\pi_{2}$-type transfer integrals.

The Coulomb interaction term $\mathcal{H}_{\text {int }}$ consists of the four components as

$$
\mathcal{H}_{\text {int }}=\mathcal{H}_{U}+\mathcal{H}_{U^{\prime}}+\mathcal{H}_{J}+\mathcal{H}_{J^{\prime}}
$$

where

$$
\begin{aligned}
\mathcal{H}_{U} & =U \sum_{i} \sum_{\gamma} n_{i \gamma \uparrow} n_{i \gamma \downarrow}, \\
\mathcal{H}_{U^{\prime}} & =U^{\prime} \sum_{i} \sum_{\gamma>\gamma^{\prime}} n_{i \gamma} n_{i \gamma^{\prime}} \\
\mathcal{H}_{J} & =J \sum_{i} \sum_{\gamma>\gamma^{\prime}} \sum_{\tau, \tau^{\prime}} c_{i \gamma \tau}^{\dagger} c_{i \gamma^{\prime} \tau} c_{i \gamma^{\prime} \tau^{\prime}}^{\dagger} c_{i \gamma \tau^{\prime}} \\
\mathcal{H}_{J^{\prime}} & =J^{\prime} \sum_{i} \sum_{\gamma \neq \gamma^{\prime}} c_{i \gamma \uparrow}^{\dagger} c_{i \gamma^{\prime} \uparrow} c_{i \gamma \downarrow}^{\dagger} c_{i \gamma^{\prime} \downarrow} .
\end{aligned}
$$


Here, $\mathcal{H}_{U}, \mathcal{H}_{U^{\prime}}, \mathcal{H}_{J}$ and $\mathcal{H}_{J^{\prime}}$ denote the intra- and inter-orbital Coulomb interactions, the exchange interaction and the pair-hopping term, respectively. The density operators are defined by $n_{i \gamma \tau}=c_{i \gamma \tau}^{\dagger} c_{i \gamma \tau}$ and $n_{i \gamma}=\sum_{\tau} n_{i \gamma \tau}$.

The third term in eq. (1), $\mathcal{H}_{\text {trig }}$, represents the effect of the trigonal distortion of $\mathrm{VO}_{6}$ octahedra. The lattice structure determined by experiments indicates that the $\mathrm{VO}_{6}$ octahedron including the V2 site is substantially compressed in the [111] direction. ${ }^{10}$ The trigonal distortion leads to the crystal-field splitting of three-fold $t_{2 g}$ levels at the $\mathrm{V} 2$ site into the low-energy $a_{1 g}$ singlet and the high-energy $e_{g}$ doublet. This crystal-field effect is described by

$$
\mathcal{H}_{\text {trig }}=-\frac{D}{2} \sum_{\gamma>\gamma^{\prime}} \sum_{\tau}\left(c_{0 \gamma \tau}^{\dagger} c_{0 \gamma^{\prime} \tau}+\text { H.c. }\right) \text {. }
$$

The compression of $\mathrm{VO}_{6}$ corresponds to a positive $D$, and the energy splitting between the $a_{1 g}$ singlet and the $e_{g}$ doublet is $3 D / 2$ in this definition. We neglect small distortions of octahedra including V3 sites for simplicity.

The multiorbital Hubbard model given by eq. (1) is too complicated to fully handle its electronic state, but we can make a simplification on the basis of the lattice structure determined by the experiment. The key observation is that the length of the V3-V3 bonds is considerably shorter than that of V2-V3 bonds. In fact, the V3-V3 bonds in the heptamer are the shortest ones in the original pyrochlore system, constituting the trimers in the Kagomé layers. Hence, we expect that the $\sigma$-type transfer integrals for these V3-V3 bonds are the most dominant among various contributions in $t_{i j}^{\gamma \gamma^{\prime}}$. From this observation, we assume that the $\sigma$-type bonding state for each V3-V3 bond has a sufficiently low energy and is fully occupied by two $d$ electrons per bonding state, forming a singlet. For example, the site $i=1$ is on two V3-V3 bonds $(i, j)=(1,2)$ and $(1,3)$ which lie in the $y z$ and $z x$ planes, respectively, and therefore, the $d_{y z}$ and $d_{z x}$ orbitals constitute the bonding levels. This is indeed the case in the noninteracting limit $\mathcal{H}_{\text {int }}=0$, and is supposed to be valid in the weak-correlation regime. The interorbital interactions at V3 sites will be taken into account within the Hartree approximation [see eq. (14)].

The assumption of the dominant $\sigma$-type V3-V3 bonds allows us to simplify the model defined by eq. (1) in the following points. (i) Since there are six V3-V3 bonds in the heptamer, $6 \times 2=12$ electrons occupy the $\sigma$-type bonding states. These 12 electrons become inactive on the $\sigma$-type V3-V3 bonds. Because we have in a total of 18 electrons in the model given by eq. (1), we will consider the remaining 6 electrons in the following calculations. Hereafter, we call the orbitals constituting the $\sigma$-type V3-V3 bonds inactive orbitals and the remaining orbitals active orbitals. (ii) All $\pi_{2}$-type transfer integrals [Fig. 2(e)] can be neglected, because orbitals which are connected to active orbitals through $\pi_{2}$-type bonds are always inactive. (iii) $\pi_{1}$-type transfer integrals for V2-V3 bonds can also be neglected on the basis of a reason similar to that in item (ii). As a consequence of these simplifications, among the transfer 
integrals, only two contributions are relevant; one is the $\sigma$-type transfer integral for V2-V3 bonds, which is denoted by $t_{\mathrm{V} 2-\mathrm{V} 3}^{\sigma}$, and the other is the $\pi_{1}$-type one for $\mathrm{V} 3-\mathrm{V} 3$ bonds, $t_{\mathrm{V} 3-\mathrm{V} 3}^{\pi}$.

Consequently, on the basis of the above assumption, the Hamiltonian given by eq. (1) is simplified into the form

$$
\mathcal{H}=\tilde{\mathcal{H}}_{\text {kin }}+\tilde{\mathcal{H}}_{\text {int }}+\mathcal{H}_{\text {trig }} .
$$

The kinetic term for the remaining 6 electrons, $\tilde{\mathcal{H}}_{\text {kin }}$, is given by

$$
\tilde{\mathcal{H}}_{\text {kin }}=\mathcal{H}_{\text {kin }}^{\mathrm{V} 2-\mathrm{V} 3}+\mathcal{H}_{\text {kin }}^{\mathrm{V} 3-\mathrm{V} 3},
$$

where $\mathcal{H}_{\text {kin }}^{\mathrm{V} 2-\mathrm{V} 3}$ denotes the $\sigma$-type hoppings between the V2 site $(i=0)$ and V3 sites $(i=1-6)$, and $\mathcal{H}_{\text {kin }}^{\mathrm{V} 3-\mathrm{V} 3}$ denotes the $\pi_{1}$-type hoppings between the nearest-neighbor V3 sites:

$$
\begin{aligned}
& \mathcal{H}_{\mathrm{kin}}^{\mathrm{V} 2-\mathrm{V} 3}=-t_{\mathrm{V} 2-\mathrm{V} 3}^{\sigma} \sum_{i=1}^{6} \sum_{\tau}\left(c_{0 \alpha_{i} \tau}^{\dagger} c_{i \alpha_{i} \tau}+\text { H.c }\right), \\
& \mathcal{H}_{\mathrm{kin}}^{\mathrm{V} 3-\mathrm{V} 3}=-t_{\mathrm{V} 3-\mathrm{V} 3}^{\pi} \sum_{\langle i j\rangle \in \mathrm{V} 3} \sum_{\tau}\left(c_{i \alpha_{i} \tau}^{\dagger} c_{j \alpha_{j} \tau}+\text { H.c. }\right) .
\end{aligned}
$$

Here, $\alpha_{i}$ denotes the active orbital at the $i$-th V3 sites [for instance, $\alpha_{1}=1\left(d_{x y}\right)$ ]. Note that in eq. (11), the orbital $\alpha_{i}$ constitutes the $\sigma$-type bond between 0 -th and $i$-th sites, while the orbitals $\alpha_{i}$ and $\alpha_{j}$ at neighboring V3 sites constitute the $\pi_{1}$-type bond in eq. (12) where the summation is taken over the nearest-neighbor V3 sites. The Coulomb interaction term in eq. (9) is written as

$$
\tilde{\mathcal{H}}_{\text {int }}=\mathcal{H}_{\text {int }}^{\mathrm{V} 2}+\mathcal{H}_{\text {int }}^{\mathrm{V} 3}
$$

The former term is for the V2 site, given by the terms in eq. (3) for $i=0$ only. The latter is for the V3 sites, which is reduced into

$$
\mathcal{H}_{\mathrm{int}}^{\mathrm{V} 3}=U \sum_{i=1}^{6} n_{i \alpha_{i} \uparrow} n_{i \alpha_{i} \downarrow}+\left(2 U^{\prime}-J\right) \sum_{i=1}^{6} n_{i \alpha_{i}},
$$

where $\alpha_{i}$ denotes the active orbital as in eqs. (11) and (12). Here, the second term is the Hartree potential from electrons in the $\sigma$-type bonding states assumed for the V3-V3 bonds.

\section{Noninteracting Case: Two Different Scenarios for Singlet Formation}

Before going into the numerical study of the electronic state of the heptamer model given by eq. (9), here we consider the noninteracting case, i.e., $\tilde{\mathcal{H}}_{\text {int }}=0\left(U=U^{\prime}=J=J^{\prime}=0\right)$. In this case, we have three parameters $t_{\mathrm{V} 2-\mathrm{V} 3}^{\sigma}, t_{\mathrm{V} 3-\mathrm{V} 3}^{\pi}$ and $D$.

There are two important cases in this parameter space to consider the singlet formation in the heptamer. One is $t_{\mathrm{V} 2 \text {-V } 3}^{\sigma} \neq 0$ and $t_{\mathrm{V} 3-\mathrm{V} 3}^{\pi}=D=0$. The $\sigma$-type transfer integrals in an orbital sector ( say $d_{x y}$ ) have finite matrix elements on one straight V3-V2-V3 bond lying in the corresponding plane (in the $x y$ plane). Hence, in this case, three-site clusters are formed on straight V3-V2-V3 bonds in each orbital sector, for instance, a cluster with the sites $i=1,0,4$ 


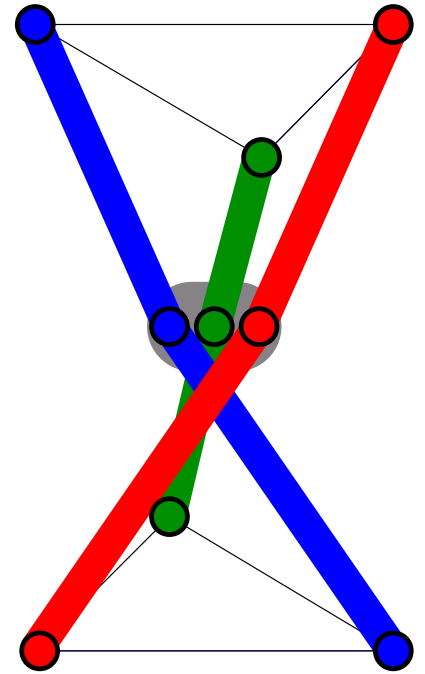

(a)

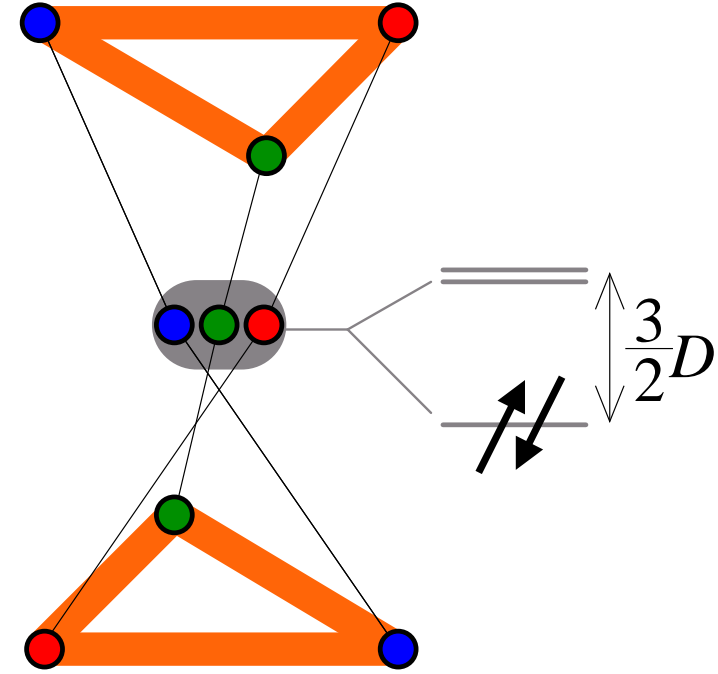

(b)

Fig. 3. Schematic pictures of two different singlet states in the noninteracting case, (a) $\sigma$-singlet state [eq. (15)] and (b) $\pi$-singlet state [eq. (16)]. Blue, red and green circles represent $d_{x y}, d_{y z}$ and $d_{z x}$ orbitals at each V site, respectively. Singlet bonds are shown by bold lines. In (b), at the V2 site, two electrons occupy the $a_{1 g}$ singlet state due to the trigonal splitting.

in the $d_{x y}$ orbital sector. In each cluster, the ground state is given by the bonding state of three sites, being singlet as shown in Fig. 3(a). The ground-state wave function for the heptamer is given by

$$
|\sigma\rangle=\prod_{\tau} \prod_{i=1}^{3} \frac{1}{2}\left(c_{i \alpha_{i} \tau}^{\dagger}+\sqrt{2} c_{0 \alpha_{i} \tau}^{\dagger}+c_{(i+3) \alpha_{i} \tau}^{\dagger}\right)|0\rangle,
$$

where $|0\rangle$ is the vacuum state of the model in eq. (9). We call this the $\sigma$-singlet state. Note that the state in eq. (15) is obtained for both positive and negative $t_{\mathrm{V} 2-\mathrm{V} 3}^{\sigma}$.

The other important case is $t_{\mathrm{V} 2-\mathrm{V} 3}^{\sigma}=0$ and $t_{\mathrm{V} 3-\mathrm{V} 3}^{\pi}, D>0$. In this case, the heptamer is separated into the isolated V2 site and two trimers with V3 sites. In each trimer, the ground state is given by the bonding state due to the $\pi_{1}$-type transfer integrals, $t_{\mathrm{V} 3 \text {-V3 }}^{\pi}$. At the V2 site, for a positive $D$, the ground state is given by a doubly occupied $a_{1 g}$ singlet as shown in Fig. 3(b). Hence, the wave function for the heptamer is expressed as

$$
|\pi\rangle=\prod_{\tau} \sum_{\gamma=1}^{3} \frac{c_{0 \gamma \tau}^{\dagger}}{\sqrt{3}} \sum_{i=1}^{3} \frac{c_{i \alpha_{i} \tau}^{\dagger}}{\sqrt{3}} \sum_{i=4}^{6} \frac{c_{i \alpha_{i} \tau}^{\dagger}}{\sqrt{3}}|0\rangle .
$$

This state is schematically shown in Fig. 3(b). We call this the $\pi$-singlet state. Note that if $t_{\mathrm{V} 3-\mathrm{V} 3}^{\pi}$ or $D$ is negative, the ground state no longer becomes singlet and has some degeneracy.

When we switch on the interactions, it is nontrivial that the ground state is singlet. Nevertheless, as we will see in the numerical results in the next section, there appear two different singlet regions; one includes the $\sigma$-singlet limit and the other includes the $\pi$-singlet 
one. In other words, in each region, the wave function is adiabatically connected with either $|\sigma\rangle$ or $|\pi\rangle$. Besides these two singlet regions, there appears also a doublet nonmagnetic ground state. Thus, our questions are twofold. (i) For realistic values of parameters, does the ground state of the heptamer Hamiltonian become singlet? (ii) If that is the case, which category does the singlet state belong to, the $|\sigma\rangle$ - or $|\pi\rangle$-like state?

\section{Effects of Electron Correlation: Exact Diagonalization Study}

\subsection{Method and parameters}

We investigate the electronic state of the model in eq. (9) including the interaction terms by exact diagonalization (ED). We use the Householder method for the ED calculations.

For the $d$ - $d$ transfer integrals, the Slater-Koster scheme ${ }^{11}$ gives the estimates $t_{\mathrm{V} 2 \text {-V } 3}^{\sigma} \simeq$ $0.5 \mathrm{eV}$ and $t_{\mathrm{V} 3-\mathrm{V} 3}^{\pi} \simeq-0.26 \mathrm{eV}$ on the basis of using the bond lengths in experiments. Hereafter,

we set $t_{\mathrm{V} 2-\mathrm{V} 3}^{\sigma}=1$ as an energy unit, and change $t_{\mathrm{V} 3-\mathrm{V} 3}^{\pi}$ from -2 to 2 and $D$ from 0 to 2 to study overall features in the parameter space, which is supposed to include realistic values for the present $t_{2 g}$ system.

Concerning the interaction parameters, no experimental estimate for $\mathrm{AlV}_{2} \mathrm{O}_{4}$ is available yet. An estimate was given for a related spinel vanadate $\mathrm{LiV}_{2} \mathrm{O}_{4},{ }^{12}$ the optical measurement suggests $U \gtrsim 2 \mathrm{eV}$. In the following, we present the results for $U=0-8$ to show the tendency with changing $U$. We set $J=0.1 U$, and retain the relations $U=U^{\prime}+2 J$ and $J=J^{\prime}{ }^{13}$

\subsection{Results}

First, we study the magnetism and the degeneracy of the ground state of the heptamer Hamiltonian given by eq. (9). Figure 4 shows the results in the $t_{\mathrm{V} 3-\mathrm{V} 3}^{\pi}-D$ plane for different values of $U$. Nonmagnetic ground states are obtained in three regions in the present parameter space; two singlet regions without any degeneracy (red and blue) and a doublet region (green). Two singlet regions are well separated, and each region includes one of the limits in the noninteracting case discussed in $\S 3$; the red region extends from the $\sigma$-singlet limit $\left(t_{\mathrm{V} 3 \text {-V3 }}^{\pi}=\right.$ $D=U=0)$, the blue region continues to the $\pi$-singlet limit $\left(t_{\mathrm{V} 2-\mathrm{V} 3}^{\sigma}=U=0\right.$ and $t_{\mathrm{V} 3-\mathrm{V} 3}^{\pi}, D>$ 0 ). The doublet region emerges only for finite values of $U$. Both singlet regions shrink as $U$ increases, but remain well for intermediate or rather large values of $U$, while the doublet region spreads as $U$ increases.

We focus on two singlet regions, one of which is believe to be relevant to understanding the experiments in $\mathrm{AlV}_{2} \mathrm{O}_{4}$ as discussed below. We will discuss the doublet region later. Although these two singlet regions are separated in the parameter space, the ground states for two regions show the same symmetry. The symmetry of the ground state of the model given by eq. (9) belongs to one of the irreducible representations $A_{1}, A_{2}$ and $E$ because the Hamiltonian belongs to the $D_{3}$ point group. ${ }^{15}$ We calculate $\left\langle\phi_{0}\left|C_{3}\right| \phi_{0}\right\rangle$ and $\left\langle\phi_{0}\left|C_{2}^{\prime}\right| \phi_{0}\right\rangle$, where $C_{3}$ and $C_{2}^{\prime}$ are operators for the symmetric rotation of a threefold symmetric axis by $3 \pi / 2$ and that of a twofold symmetric axis by $\pi$, respectively. Here, $\left|\phi_{0}\right\rangle$ is the ground-state wave vector obtained 
by ED calculations. We found that $\left\langle\phi_{0}\left|C_{3}\right| \phi_{0}\right\rangle=\left\langle\phi_{0}\left|C_{2}^{\prime}\right| \phi_{0}\right\rangle=1$ for all the ED solutions without degeneracy. Namely, all the ground states in both separated singlet regions belong to the $A_{1}$ symmetry group.

To characterize two singlet regions, we calculate overlaps of the ground-state wave function with $|\sigma\rangle$ and $|\pi\rangle$ in eqs. (15) and (16), respectively. We plot the square of overlaps in Fig. 5 for typical sets of parameters. In the red region in Fig. 4, the square of the overlap with $|\sigma\rangle$, $\left\langle\sigma \mid \phi_{0}\right\rangle^{2}$, is substantial whereas $\left\langle\pi \mid \phi_{0}\right\rangle^{2}$ is negligibly small $(<0.01)$. Since $\left\langle\sigma \mid \phi_{0}\right\rangle^{2} \gg\left\langle\pi \mid \phi_{0}\right\rangle^{2}$, we call this red region the $\sigma$-singlet region. On the other hand, in the blue region in Fig. 4 , $\left\langle\pi \mid \phi_{0}\right\rangle^{2}$ has a substantial value compared with $\left\langle\sigma \mid \phi_{0}\right\rangle^{2}<0.01$, and therefore we call the blue region the $\pi$-singlet region. Hence, these two singlet regions are well distinguished by the values of overlaps, and appear to be separated within the parameter space that we investigated.

We also calculate the charge disproportionation between the V2 site and the V3 sites in the ground state. Figure 6 shows the electron density at the V2 site;

$$
N_{2}=\left\langle\phi_{0}\left|\sum_{\gamma} n_{0 \gamma}\right| \phi_{0}\right\rangle
$$

Note that the electron density at the V3 site is given by $\left(18-N_{2}\right) / 6$ for singlet states with $A_{1}$ symmetry. In the $\sigma$-singlet region (red region in Fig. 4), as $U$ increases, $N_{2}$ continuously decreases from the noninteracting value 3 . On the contrary, in the $\pi$-singlet region (blue region in Fig. 4), $N_{2}$ is almost unchanged and remains at the noninteracting value 2. This difference comes from the nature of the singlet states. In the former, since the dominant singlet bonds are on the straight V3-V2-V3 clusters, electron charges are easily redistributed between V2 and V3 sites. On the other hand, in the latter $\pi$-singlet region, the dominant singlet bonds are confined within V3 trimers, and the bonds between V2 and V3 sites are relatively weak. There, the singlet energy gain within the V3 trimers as well as the crystal field $D$ at the V2 site prevents the charge redistribution between V2 and V3 sites.

The results in Figs. 4 and 5 show that for the estimates of transfer integrals by using the Slater-Koster scheme, the ground state of the model given by eq. (9) is singlet and belongs to the $\sigma$-singlet region where the ground-state wave function is close to $|\sigma\rangle$ in eq. (15). We note that the $\pi$-singlet region is only limited for large values of $t_{\mathrm{V} 2 \text {-V3 }}^{\pi}$ and $D$ compared with $t_{\mathrm{V} 2-\mathrm{V} 3}^{\sigma}$, and this is not realistic in the present $t_{2 g}$ electron system. Therefore, we conclude that the singlet ground state is realized in the heptamer, and that the basic mechanism of singlet formation is understood on the basis of the $\sigma$-type bonding of $t_{2 g}$ orbitals.

We calculate the spin gap $\Delta$ for a comparison with experimental data, which we define here as the energy gap from the ground state to the lowest magnetic excited state. Note that the spin gap becomes zero when the ground state is magnetic by definition. The result is shown in Fig. 7 for several Slater-Koster parameters. Although a quantitative comparison with the experimental estimate is beyond the scope of the present study since interactions among the 
heptamers are completely neglected in the present theory, ${ }^{16}$ this finite spin gap gives rise to the suppression of magnetic susceptibility below $T_{c}$ observed in experiments. Therefore, our results on the gapped, spin-singlet ground state in the heptamer model support the heptamer picture, in which the peculiar temperature dependence of the magnetic susceptibility is explained as the summation of the heptamer-singlet contribution and the $S=1$ Curie-like contribution. ${ }^{10}$

Finally, let us make a remark on the doublet state (green region) in Fig. 4. The doublet region develops as $U$ increases, and includes the parameter regime given by the Slater-Koster scheme for large values of $U>8$. From the symmetry analysis, we find that the doublet state belongs to $E$ symmetry. Since this state is also nonmagnetic, it might be possible to explain the peculiar temperature dependence of the magnetic susceptibility. Nevertheless, we believe that the $\sigma$-type singlet ground state is relevant to $\mathrm{AlV}_{2} \mathrm{O}_{4}$ for the following reasons. One is that the degeneracy remaining in the doublet state should be lifted by the generalized Jahn-Teller mechanism or the heptamer-heptamer interaction when we allow a further lattice distortion of the heptamer; however, such lowering of the symmetry is not observed in experiments. The other reason is that our present analyses are justified in the weak correlation regime as discussed in $\S 2$, and hence some effects beyond our assumptions may alter the results in the relatively large $U$ region.

\section{Summary and Concluding Remarks}

We have investigated the origin of spin-singlet formation in seven-site clusters, heptamers, which emerge in the spinel oxide $\mathrm{AlV}_{2} \mathrm{O}_{4}$ as a result of the structural change with the trimer formation in Kagomé layers of the $\mathrm{V}$ pyrochlore lattice. We have derived an effective model to describe the charge, spin and orbital degrees of freedom in one heptamer from the Hubbard

model with $t_{2 g}$ orbital degeneracy, and investigated its electronic and magnetic states using numerical exact diagonalization. Our results indicate that in the realistic parameter region for $\mathrm{AlV}_{2} \mathrm{O}_{4}$, the ground state of the heptamer becomes spin-singlet, and that not the trimer formation in the Kagomé layers but the heptamer formation plays an essential role in the spin-singlet formation in this system. We conclude that the singlet state is stabilized by dominant $d$ - $d$ transfer integrals of the $\sigma$-type which form bonding states within the heptamer. The heptamer formation can give a comprehensive understanding of the magnetic properties observed in experiments; a suppression due to the singlet gap formation and the Curie-like behavior at lower temperatures coming from $S=1$ moments outside the heptamers.

How can we distinguish experimentally the three different origins of spin-singlet formation, i.e., two different singlet states ( $\sigma$ - and $\pi$-type) and one doublet state? We can distinguish between singlet and doublet states by the entropy at high temperatures estimated from the electronic specific heat. Then, it is possible to distinguish between $\sigma$ - and $\pi$-type singlet states which have the same symmetry. One possible way is to detect the directional dependence of the spin excitation. The dominant singlet bonds lie on the intertrimer V3-V2-V3 bonds in the 
$\sigma$ case and within the V3 trimers on Kagomé planes in the $\pi$ case. This difference may be detected using the Raman scattering technique. Another possibility is to measure the local density of $d$ electrons at the V2 site. Our results in Fig. 6(f) indicate that the local density becomes $\sim 2.5-3.0$ in the $\sigma$-singlet region, while it is $\simeq 2.0$ in the $\pi$-singlet one.

Let us comment on the properties of $S=1$ moments outside the heptamers. In our heptamer scenario, below $T_{\mathrm{c}}$, the magnetic moments that remain active are the $S=1$ spins at V1 sites only, and the spins at V2 and V3 sites are magnetically inactive, because of the singlet formation in heptamers. Since a V1 site is connected to the V2 sites (see Fig. 1), the V1 spins are almost magnetically isolated. At a much lower temperature than $T_{\mathrm{c}}$, another anomaly appears at $T \sim 5 \mathrm{~K}$ in the magnetic susceptibility, which is ascribed to spin-glass transition. ${ }^{8}$ This indicates that in this low-temperature region, $S=1$ spins begin to interact with each other. Nearest-neighbor V1 pairs correspond to the third-nearest-neighbor sites across hexagons in the original pyrochlore lattice, forming a two-dimensional triangular lattice in the [111] planes. Hence, the $S=1$ moments at V1 sites constitute a weakly coupled triangular spin system at low temperatures. The sign of exchange interactions is not trivial because of complicated exchange paths, but in any case, it is believed that Heisenberg spin systems on a weakly coupled triangular lattice show a magnetic order, not a spin-glass behavior. Therefore, our heptamer scenario suggests that the spin-glass behavior comes from an extrinsic effect, such as randomness.

Our present study was conducted on the basis of the lattice structure below the structural transition temperature, and therefore, at this stage, we cannot answer why and how the lattice distortion including the formation of heptamers is stabilized in the frustrated pyrochlore lattice structure. There are many similar examples of such clusters in frustrated systems, such as

hexamers in $\mathrm{ZnCr}_{2} \mathrm{O}_{4},{ }^{17}$ octamers in $\mathrm{CuIr}_{2} \mathrm{~S}_{4},{ }^{5,18}$ trimers in $\mathrm{LiVO}_{2},{ }^{19}$ and dodecamers in a double-exchange spin-ice model on a Kagomé lattice. ${ }^{20}$ These facts suggest the ubiquitous role of cluster formations in lifting the degeneracy inherent in frustrated systems, although the driving force may depend on the details of systems. In the present system $\mathrm{AlV}_{2} \mathrm{O}_{4}$, our results imply that the stability of the heptamer singlet state itself may play some role in the mechanism of self-organizing the clusters. However, to identify a dominant player, it is necessary to handle charge, spin, orbital and lattice degrees of freedom and to compare the instabilities to many possible ordered states on the frustrated pyrochlore lattice. This is left for future study.

\section{Acknowledgment}

We would like to thank M. Shingu, T. Katsufuji, S. Mori and Y. Horibe for stimulating discussions. This work was supported by a Grant-in-Aid for the 21st COE program and for Scientific Research (No. 16GS50219) from the Ministry of Education, Culture, Sports, Science and Technology of Japan. 

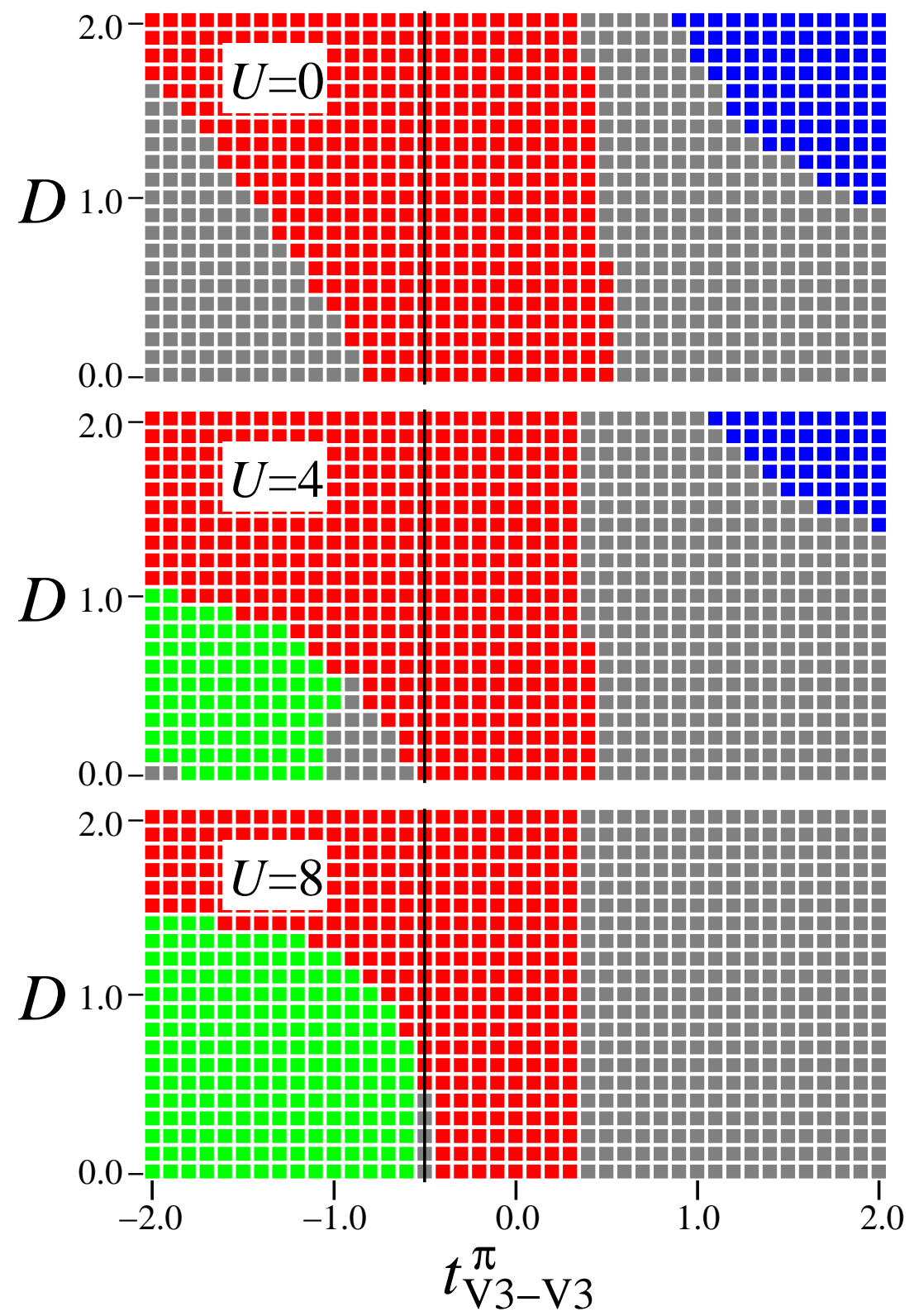

nonmagnetic $(\sigma-$ singlet $)$
nonmagnetic $(\pi-$ singlet $)$
nonmagnetic (doublet)
magnetic
- Slater-Koster parameters

Fig. 4. Magnetism of the ground state of the heptamer model for several $U$. The red, blue and green regions denote the $\sigma, \pi$-singlet and doublet regions, respectively. See the text for details. 


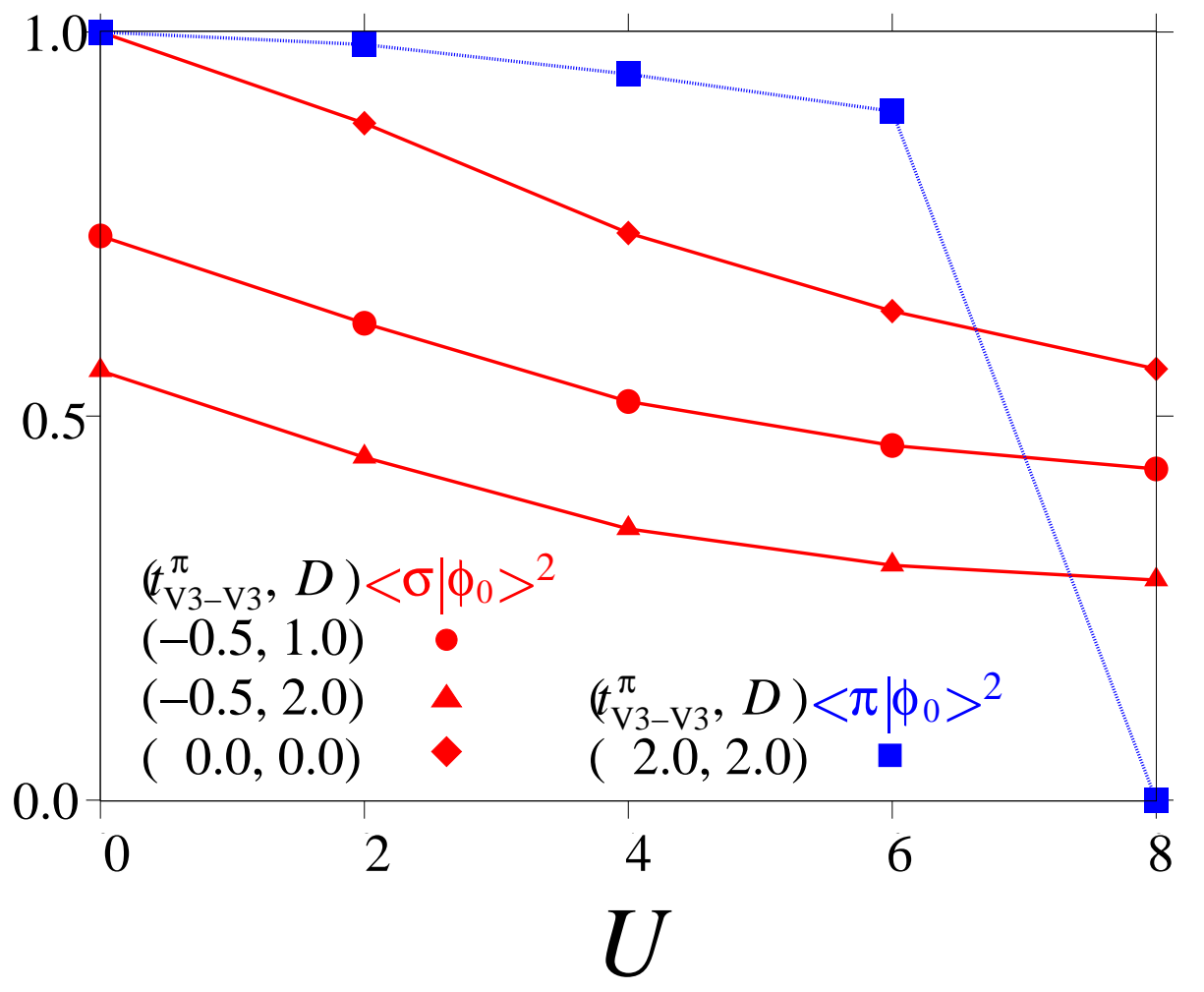

Fig. 5. Square of overlap $\left\langle\sigma \mid \phi_{0}\right\rangle^{2}$ and $\left\langle\pi \mid \phi_{0}\right\rangle^{2}$ for several sets of $\left(t_{\mathrm{V} 3-\mathrm{V} 3}^{\pi}, D\right)$. The solid lines are guides for the eyes. 
(a)
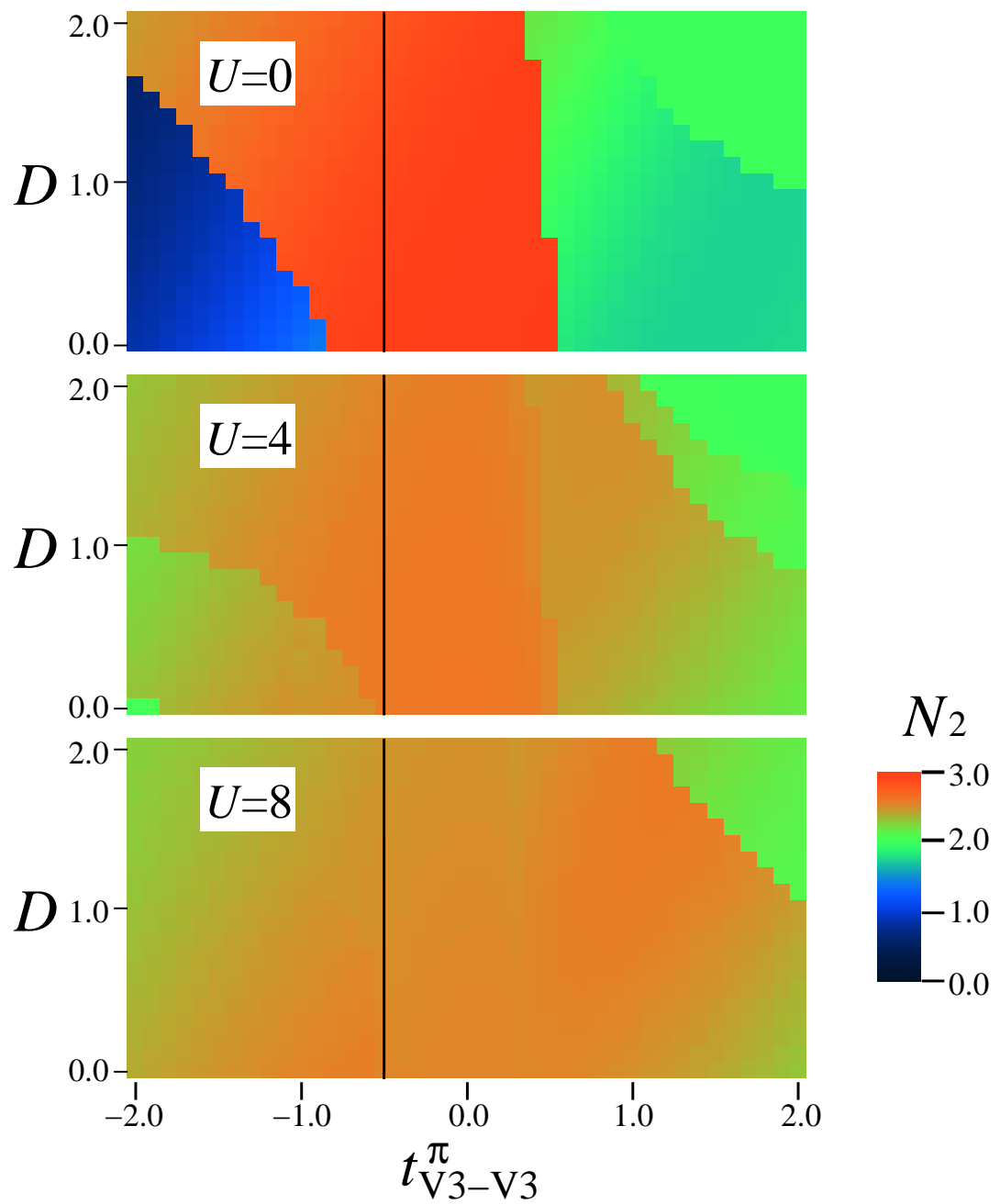

\section{- Slater-Koster parameters}

(b)

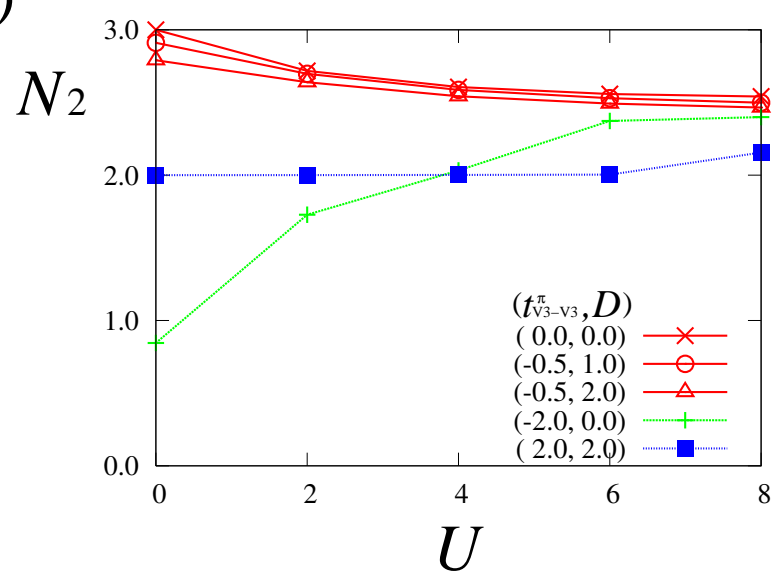

Fig. 6. (a) Charge density at V2 site, $N_{2}$, for several $U$. In the degenerate regions, we plot the values averaged over all degenerate states. $U$ dependences are shown in (b) for several sets of $\left(t_{\mathrm{V} 3-\mathrm{V} 3}^{\pi}, D\right)$. The solid lines in (b) are guides for the eyes. 


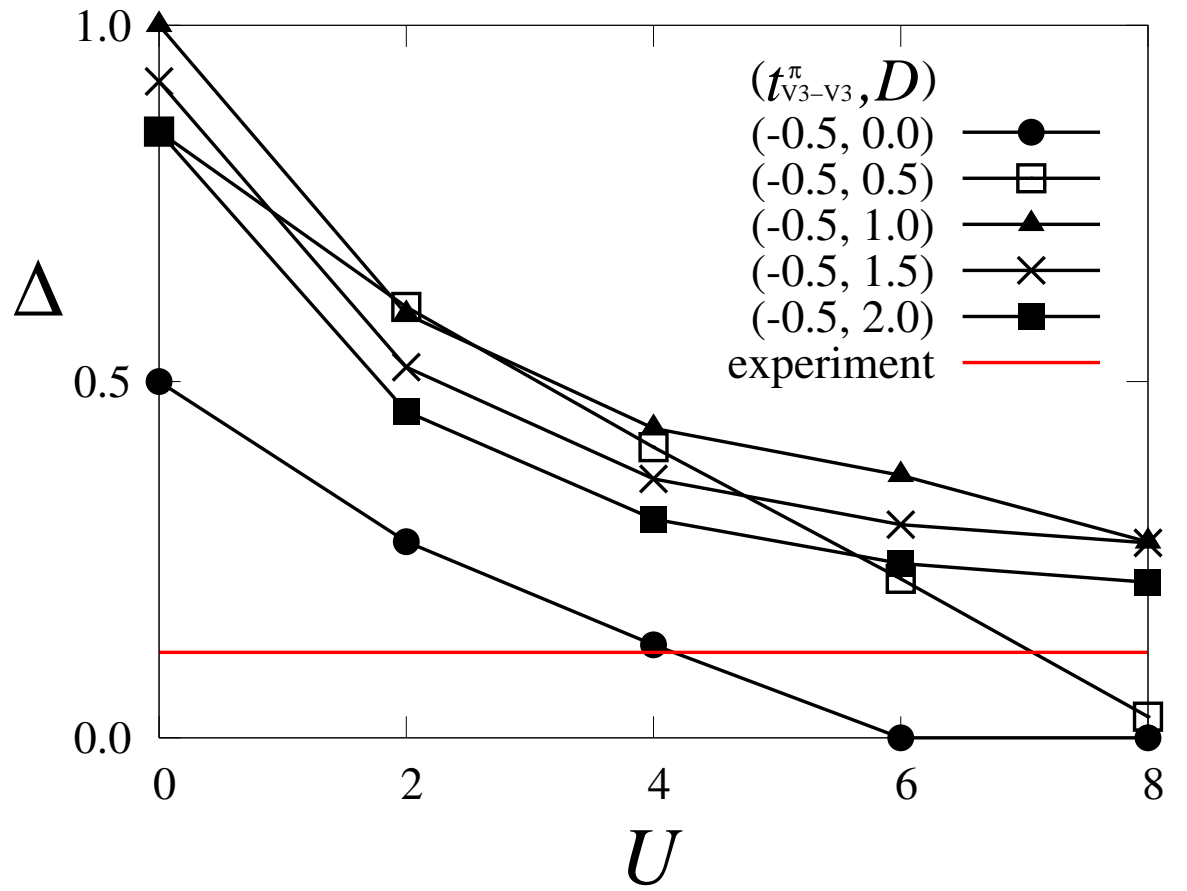

Fig. 7. Spin gap for the heptamer model given by eq. (9) for several Slater-Koster parameters $\left(t_{\mathrm{V} 3-\mathrm{V} 3}^{\pi}, D\right)$. 


\section{References}

1) Magnetic System with Competing Interaction, ed. by H. T. Diep (World Scientific Publishing Co. Pte. Ltd., Singapore, 1994).

2) R. Liebmann, Statistical Mechanics of Periodic Frustrated Ising Systems (Springer-Verlag, Berlin, Tokyo, 1986).

3) P. W. Anderson: Phys. Rev. 102 (1956) 1008.

4) E. J. W. Verwey: Nature (London) 144 (1939) 327.

5) P. G. Radaelli, Y. Horibe, M. J. Gutmann, H. Ishibashi, C. H. Chen, R. M. Ibberson, Y. Koyama, Y. S. Hor, V. Kiryukhin and S-W. Cheong: Nature 416 (2002) 155.

6) S. Kondo, D. C. Johnston, C. A. Swenson, F. Borsa, A. V. Mahajan, L. L. Miller, T. Gu, A. I. Goldman, M. B. Maple, D. A. Gajewski, E. J. Freeman, N. R. Dilly, R. P. Dickey, J. Merrin, K. Kojima, G. M. Luke, Y. J. Uemura, O. Chmaissem and J. D. Jorgensen: Phys. Rev. Lett. 78 (1997) 3729 .

7) C. Urano, M. Nohara, S. Kondo, F. Sakai, H. Takagi, T. Shiraki and T. Okubo: Phys. Rev. Lett. 85 (2000) 1052.

8) K. Matsuno, T. Katsufuji, S. Mori, Y. Moritomo, A. Machida, E. Nishibori, M. Takata, M. Sakata, N. Yamamoto and H. Takagi: J. Phys. Soc. Jpn. 70 (2001) 1456.

9) K. Matsuno, T. Katsufuji, S. Mori, M. Nohara, A. Machida, Y. Moritomo, K. Kato, E. Nishibori, M. Takata, M. Sakata, K. Kitazawa and H. Takagi: Phys. Rev. Lett. 90 (2003) 096404.

10) Y. Horibe, M. Shingu, K. Kurushima, H. Ishibashi, N. Ikeda, K. Kato, Y. Motome, N. Furukawa, S. Mori and T. Katsufuji: Phys. Rev. Lett. 96 (2006) 086406.

11) J. C. Slater and G. F. Koster: Phys. Rev. 94 (1954) 1498.

12) A. Fujimori, K. Kawakami and N. Tsuda: Phys. Rev. B 38 (1988) 7889.

13) J. Kanamori: Prog. Theor. Phys. 30 (1963) 275.

14) J. Matsuno, A. Fujimori and L. F. Mattheiss: Phys. Rev. B 60 (1999) 1607.

15) Formally, the point group of the Hamiltonian is $D_{3 d}=D_{3} \otimes C_{i}$.

16) Another difficulty in the quantitative comparison is the spin quantum number in the magnetic excited state. The fitting of the temperature dependence of the magnetic susceptibility depends on the spin quantum number which is unknown experimentally at this stage.

17) S.-H. Lee, C. Broholm, W. Ratcliff, G. Gasparovic, T. H. Kim, Q. Huang and S-W. Cheong: Nature 418 (2002) 856.

18) D. I. Khomskii and T. Mizokawa: Phys. Rev. Lett. 94 (2005) 156402.

19) H. F. Pen, J. van den Brink, D. I. Khomskii and G. A. Sawatzky: Phys. Rev. Lett. 78 (1997) 1323.

20) Y. Shimomura, S. Miyahara and N. Furukawa: J. Phys. Soc. Jpn. 73 (2004) 1623. 